\title{
A Graphene-Based Sleep Mask for Comfortable Wearable Eye Tracking
}

DOI:

10.1109/EMBC.2019.8857198

\section{Document Version}

Accepted author manuscript

Link to publication record in Manchester Research Explorer

\section{Citation for published version (APA):}

Beach, C., Karim, M. N., \& Casson, A. (2019). A Graphene-Based Sleep Mask for Comfortable Wearable Eye Tracking. In 41st Annual International Conference of the IEEE Engineering in Medicine \& Biology Society https://doi.org/10.1109/EMBC.2019.8857198

\section{Published in:}

41st Annual International Conference of the IEEE Engineering in Medicine \& Biology Society

\section{Citing this paper}

Please note that where the full-text provided on Manchester Research Explorer is the Author Accepted Manuscript or Proof version this may differ from the final Published version. If citing, it is advised that you check and use the publisher's definitive version.

\section{General rights}

Copyright and moral rights for the publications made accessible in the Research Explorer are retained by the authors and/or other copyright owners and it is a condition of accessing publications that users recognise and abide by the legal requirements associated with these rights.

\section{Takedown policy}

If you believe that this document breaches copyright please refer to the University of Manchester's Takedown Procedures [http://man.ac.uk/04Y6Bo] or contact uml.scholarlycommunications@manchester.ac.uk providing relevant details, so we can investigate your claim.

\section{OPEN ACCESS}




\title{
A Graphene-Based Sleep Mask for Comfortable Wearable Eye Tracking
}

\author{
Christopher Beach, Student Member, IEEE, Nazmul Karim, and Alexander J. Casson, Senior Member, IEEE
}

\begin{abstract}
We present a new wearable electrooculogram (EOG) monitor for measuring eye movements. We fabricated conductive and flexible graphene-based textiles from nylon to use as a sensing electrode, which we then integrated into a commercially available eye mask held in place only with the standard elastic strap. We tested this mask on 4 participants to quantify the noise floor and show that we can detect eye blinks to a high SNR of over $16 \mathrm{~dB}$. We also identify that the material can detect other eye movements in cases when the noise floor is low. As our system is held in place with only an elastic strap it offers the same level of comfort as when wearing a normal eye mask. Our sensors offer an increased level of comfort over conventional gelled electrodes traditionally used in EOG monitoring and may be of use for comfortable eye movement experiments. This is particularly important during sleep studies where the EOG is routinely monitored, but using bulky instrumentation.
\end{abstract}

\section{INTRODUCTION}

In the UK, around $18 \%$ of adults get an insufficient amount of sleep each night [1]. Poor sleep can lead to secondary health problems such as cardiovascular disease and stroke [2]. A key stage of sleep is thought to be Rapid Eye Movement (REM) sleep [3] and achieving REM sleep is seen as quality sleep. Achieving a high amount of REM sleep often leads to the person feeling more refreshed, as well as having improved memory capabilities [3]. The gold standard for monitoring sleep is the polysomnogram (PSG), which includes the recording of electroencephalogram (EEG) (brain activity), and the recording of electrooculography (EOG) (the movement of the eyes). These PSG recordings can be analyzed by a sleep expert to provide a scoring of the quality of sleep and to classify the amount of each stage of sleep the person has [4].

PSG recording devices used in sleep studies are large, bulky and uncomfortable for the user. They require many channels, and therefore a large cable count and a high number of electrodes connected to the skin [5]. Electrodes are typically made from a gelled silver/silver chloride $(\mathrm{Ag} / \mathrm{AgCl})$ material that is stuck to the skin. These electrodes are uncomfortable for the user, and may cause skin irritation. Additionally, the electrodes dry out over time, leading to a degradation in signal quality. Further, as these electrodes

This work was supported by the UK Engineering and Physical Sciences Research Council grant number EP/N509565/1 and the Doctoral Training Partnership with the University of Manchester.

C. Beach and A. J. Casson are with the School of Electrical and Electronic Engineering, The University of Manchester, UK. Email: christopher.beach@postgrad.manchester.ac.uk, alex.casson@manchester.ac.uk.

N. Karim is with the National Graphene Institute (NGI), The University of Manchester, UK. Email: monazmul.karim@manchester.ac.uk. are tightly adhered to the skin, they can impact the quality of sleep leading to recordings that do not accurately reflect the user's natural sleep patterns. Electrodes in a PSG study typically need to be placed by an expert, reducing the ability for these recordings to be taken in a person's own home, where they would normally sleep. The PSG is widely used in sleep clinics but is impractical to set up in a home environment. Many fitness devices such as the Fitbit have emerged that monitor sleep from the user's physical activity, but are limited in accuracy. There is growing interest in accurate sleep monitoring at home with devices such as the Rythm Dreem coming on the market for at-home EEG monitoring. There are however, relatively few at-home, comfortable, EOG devices. Liang et al. [6] demonstrates a system that can be used for EOG recordings using electrodes made from commercial dry fabric sensors sewn into an eye mask. Golparvar et al. [7] further demonstrates the capabilities of using graphene-based textile sensors to take these recordings in a system that is fitted around the forehead and held on with a band.

We have previously demonstrated how graphene-based textiles can be used as a bio sensing material in electrocardiogram (ECG) recordings [8], and how they offer stable performance in real-world conditions [9]. In this work we expand this, and the graphene EOG system in [7], by showing how our method for creating graphene textiles can be used for EOG recordings sewn to a conventional eye mask, while held in place using only the standard elastic strap. This leads to a significant increase in comfort, as the mask is very similar to an unmodified eye mask, commonly worn by people as they sleep. Our sensing material has been shown to be washable in a home washing machine [10], as well as requiring less waste materials and water utilization during fabrication, creating a more environmentally friendly process. Further, we quantify the noise performance of this material by analyzing the signal-to-noise ratio (SNR) of the eye-movement features for the first time. The remainder of this paper is structured as follows. Section II describes the fabrication of the conductive material, the creation of the sleep-mask and the protocol for verifying the performance of the device. Section III reports the performance of the device by analyzing the SNR of the each of the features for a total of four participants. Finally, conclusions are drawn in Section IV.

\section{METHODS}

\section{A. Preparation of conductive graphene-based nylon}

To prepare the conductive material we used a previously reported method detailed in [10]. In conventional processes, 


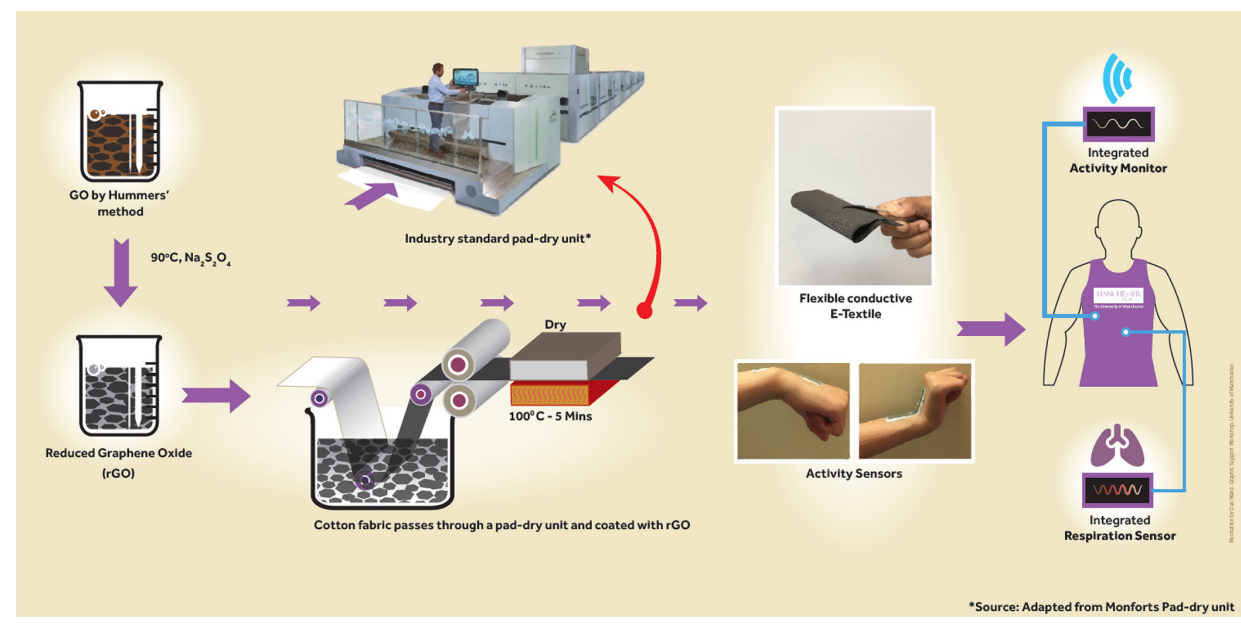

Fig. 1. Diagram of the production process to create the conductive graphene-based textile. Image reproduced from [10], with permission from ACS.

a material is coated with graphene oxide (GO) and then reduced. However, this reduction can damage the structure of the coated material. Further, this often requires the use of chemicals that are non-bio-compatible yielding an electrode that is unsuitable for use as a bio-sensing electrode.

In our process we created conductive textiles on a scoured and bleached nylon knitted fabric. The first step in this process is reducing a solution of graphene oxide, functionalizing the solution, and then coating the textile material with this. Here we used the modified Hummers' method to prepare graphene oxide (GO), which was then chemically reduced to create reduced graphene oxide ( $\mathrm{rGO}$ ) using sodium hydrosulphite $\left(\mathrm{Na}_{2} \mathrm{~S}_{2} \mathrm{O}_{4}\right) \cdot \mathrm{Na}_{2} \mathrm{~S}_{2} \mathrm{O}_{4}$ was found to be a highly effective reducing agent to achieve high electrical conductivity [11]. We functionalized the surface of rGO with poly-styrenesulfonate (PSS), this was incorporated into the solution to allow for a stable dispersion when the textile material was coated. This substance then coated the textile using an industry standard pad-dry unit, and was subsequently dried at $100{ }^{\circ} \mathrm{C}$ for five minutes. This process is summarized in Fig. 1. The resulting textile material had a sheet resistance below $40 \mathrm{k} \Omega / \mathrm{sq}$.

\section{B. Creation of eye-tracking mask}

Sleep can be classified using only two EOG channels two horizontal electrodes placed either side of the eyes and one reference electrode placed on the forehead [12]. This significantly reduces the number of cables required for a sleep study down to only three. We create our electrodes by sewing our processed nylon fabric into a standard, offthe-shelf eye mask using non-conductive polyester thread. To connect the electrodes to an amplifier, snap connectors (Prym $12 \mathrm{~mm}$ buttons) were fitted onto each textile electrode. Two masks were made to investigate the performance. The first of these masks (from here on referred to as mask A) uses electrodes with dimensions of $35 \times 20 \mathrm{~mm}$, with a total of three electrodes (two for horizontal movement and one reference) sewn in to the mask as shown in Fig. 2(a). The second mask (referred to as mask B) uses two larger electrodes of $20 \times 55 \mathrm{~mm}$ sewn either side of the eyes for

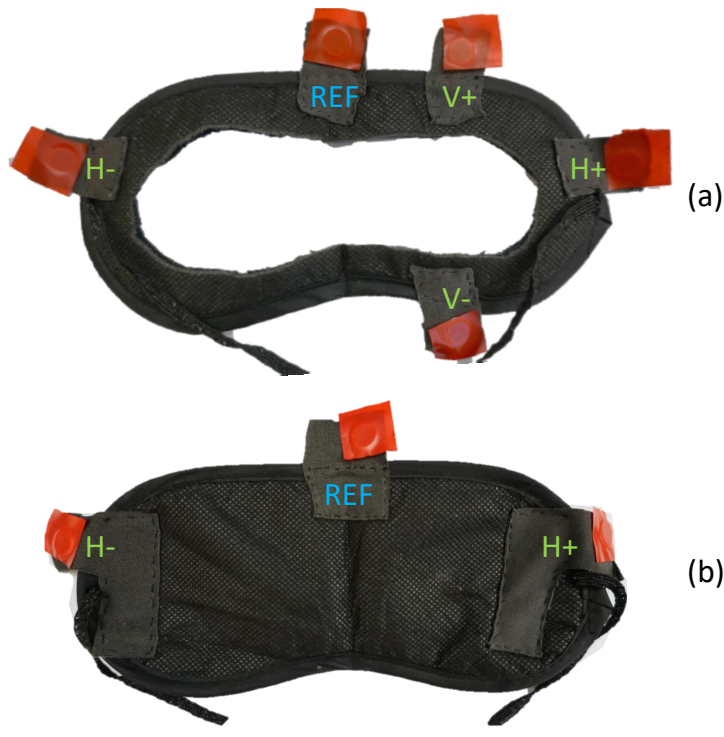

Fig. 2. Sleep masks with graphene-based nylon sensors sewn in, showing the view from the back of the mask. Electrical tape has been used to prevent the metal back of the snap connectors touching the skin. (a) Mask A (note $\mathrm{V}+$ and $\mathrm{V}$ - electrodes were not used in this paper) (b) Mask B.

horizontal movements and one smaller reference electrode of dimensions $20 \times 30 \mathrm{~mm}$. This is shown in Fig. 2(b). Mask A had a hole cut into it to allow the participant to see a screen to follow instructions given in the experiment, while the second one was left as supplied. Preliminary results found that the hole cut in mask A damaged the structure of the mask and therefore the ability of the mask to stay adhered to the participant's face during testing, therefore it was opted not to cut a hole in mask B.

\section{Experimental setup}

To verify our device, an eye-tracking experiment was created on a PC using Unity. The program instructed the user to perform a series of voluntary blinks, as well as smooth pursuit eye movements that were performed by instructing the user to follow a moving ball on the screen with their eyes. The software also handled the timing of each of these actions to allow repeatable measurements between participants. 
The full experimental protocol is detailed in Table I. This involved movements right (dextroversion), left (levoversion), up (supraversion) and down (infraversion), with and without fixations as well as voluntary blinks. Participants also read a passage of text. Note that only the movements highlighted in green and blue in Table I are analyzed in this paper. To undertake the experiment, participants were asked to sit facing a computer screen, placed at a $30 \mathrm{~cm}$ distance from their eyes, and to follow the protocol. Participants had a run through of the experiment to allow familiarization with the protocol before any signals were recorded, and they were also asked to reduce involuntary blinks and any other undesired eye movements as much as possible. The experiment was repeated for both of the masks. For mask B, the instructions were read out to the participants as they were unable to see the computer screen through the sleep mask.

TABLE I

PROTOCOL FOR VERIFYING THE PERFORMANCE OF THE EYE MASK, TIME CODES $\left(t_{0}\right.$ ETC.) ARE USED TO REFER TO EACH ACTIVITY.

\begin{tabular}{cc}
\hline Time & Movement \\
\hline$t_{0}=0 \mathrm{~s}$ & Experiment start \\
$t_{1}=5 \mathrm{~s}$ & Blink \\
$t_{2}=10 \mathrm{~s}$ & Smooth dextroversion \& fixation \\
$t_{3}=20 \mathrm{~s}$ & Smooth levoversion \& fixation \\
$t_{4}=40 \mathrm{~s}$ & Blink \\
$t_{5}=45 \mathrm{~s}$ & Smooth dextroversion, levoversion \& return to centre \\
$t_{6}=55 \mathrm{~s}$ & Blink \\
$t_{7}=60 \mathrm{~s}$ & Smooth supraversion, infraversion \& return to centre \\
$t_{8}=70 \mathrm{~s}$ & Blink \\
$t_{9}=75 \mathrm{~s}$ & Passage reading \\
$t_{10}=110 \mathrm{~s}$ & Blink \\
\hline
\end{tabular}

We recruited four male participants to undertake this experiment. All procedures were approved by the University of Manchester Research Ethics Committee, application number 2018-4015-5619. The device was connected to a standard biomedical amplifier (Actiwave EEG/ECG 4 channel, CamNtech), which was set to record in EDF format sampling at $1024 \mathrm{~Hz}$ at 10 bits, using the ECG gain setting.

\section{Analysis methods}

Prior to analysis the data was filtered with an eighth-order zero-phase bandpass filter with $f_{L}=10 \mathrm{~Hz}$ and $f_{H}=1 \mathrm{~Hz}$. The low pass filtering removes the large amount of $50 \mathrm{~Hz}$ interference on the signal due to the mains line noise coupling to the body, while the high-pass filtering removes baseline wander. The data from the negative horizontal electrode was then subtracted from the positive horizontal electrode to make the final signal. The first voluntary blink in the experiment was used for synchronization purposes, and the data up to and including this point was not included in the analysis.

The timings of each activity detailed in Table I were manually retrieved in each record for analysis and any sections with large amounts of motion artifacts and nonvoluntary blinks were not discarded. This can lead to a lower reported SNR, but more accurately reflects the real-world performance of the device where unintended movements and

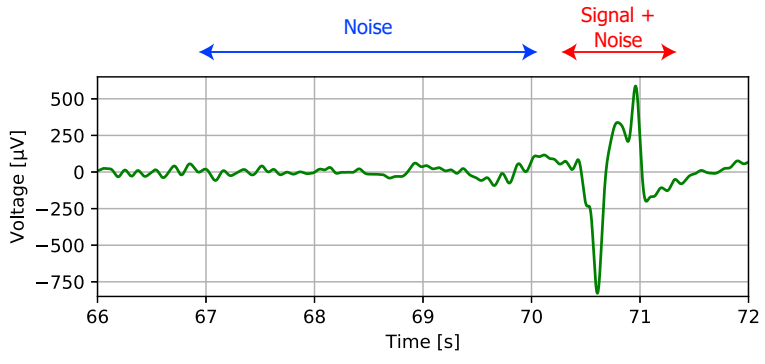

Fig. 3. Sample data from one record showing the calculation of SNR for an eye blink using mask B. The period used to calculate the noise as well as the signal + noise section are shown.

other interference may be present. We calculate the SNR for each of the eye blinks using (1). To calculate the noise ( $N$ or $N_{r m s}^{a v g}$ ), the Root Mean Square (RMS) of the $3 \mathrm{~s}$ of data before the action was calculated, allowing a $0.5 \mathrm{~s}$ gap between the end of the noise and the action starting. This is illustrated in Fig. 3. Given the small amplitude signals in EOG recordings, it is not appropriate to assume that taking the RMS of the window when the eye movement is happening will give an accurate representation of the signal $(S)$, as the amplitude of the signal is not necessarily significantly larger than the noise floor. Instead, we measure the RMS of the signal-plus-noise, which we refer to as $S+N_{r m s}^{\text {embedded }}$ and subtract the average RMS noise of the previous section $\left(N_{r m s}^{a v g}\right)$ to provide an estimate for the RMS value of $S$.

$$
S N R=\left(\frac{S}{N}\right)=\left(\frac{\left(S_{r m s}+N_{r m s}^{e m b e d d e d}\right)-N_{r m s}^{a v g}}{N_{r m s}^{a v g}}\right)
$$

\section{RESULTS}

\section{A. Measuring SNR of eye blinks}

The SNR for detecting each of the eye blinks is shown in Table II. Note that there are a few events where the SNR could not be calculated as a result of the estimation we make in (1) which can give a negative SNR value, making it not possible to calculate a SNR value in $\mathrm{dB}$. These entries have been entered into the table as NaN. Generally such cases occurred due to an unintended movement or similar being present during the analysis window.

For mask $\mathrm{A}$ it can be seen that the average SNR for detecting an eye blink is over $10 \mathrm{~dB}$, while with mask B it is much higher at around $16.5 \mathrm{~dB}$. The increase in performance with mask B is a result of using larger electrodes, increasing the chance of a good connection with the skin. Further, the fact that mask B did not have a hole cut in the centre allows it to make a better connection with the face of the wearer, further increasing the quality of the connection with the skin. Nevertheless, for both masks the SNR is high and therefore the eye blinks can be easily detected.

\section{B. Detecting eye movements}

Detecting the smooth pursuit eye movements undertaken during $t_{5}$ (see Table I), when the participants gazed right, left and back to the centre was difficult with both masks. A sample record using mask B is shown in Fig. 4 for both 
TABLE II

MEASUREMENTS OF SNR FOR EACH OF THE EYE BLINK MOVEMENTS.

\begin{tabular}{|c|c|c|c|c|c|c|c|}
\hline \multirow{2}{*}{ Mask } & \multirow{2}{*}{ Activity } & \multicolumn{4}{|c|}{ SNR [dB] } & \multicolumn{2}{|c|}{ Avg. SNR [dB] } \\
\hline & & Participant 1 & Participant 2 & Participant 3 & Participant 4 & Per Activity & Across Records \\
\hline \multirow{4}{*}{ Mask A } & $2^{\text {nd }}$ Blink $\left(t_{4}\right)$ & 18.84 & $\mathrm{NaN}$ & $\mathrm{NaN}$ & 17.52 & $11.74 \pm 13.91$ & \multirow{4}{*}{$10.09 \pm 9.76$} \\
\hline & $3^{\text {rd }}$ Blink $\left(t_{6}\right)$ & $\begin{array}{r}3.98 \\
\end{array}$ & -9.08 & 9.05 & 15.71 & $8.68 \pm 7.87$ & \\
\hline & $4^{\text {th }}$ Blink $\left(t_{8}\right)$ & 0.66 & 2.12 & 8.82 & 15.55 & $8.87 \pm 7.12$ & \\
\hline & $5^{\text {th }}$ Blink $\left(t_{10}\right)$ & 18.77 & 10.01 & -0.19 & -0.99 & $10.70 \pm 11.25$ & \\
\hline \multirow{4}{*}{ Mask B } & $2^{\text {nd }}$ Blink $\left(t_{4}\right)$ & 21.00 & 3.53 & 18.55 & 13.34 & $16.20 \pm 12.59$ & \multirow{4}{*}{$16.49 \pm 15.74$} \\
\hline & $3^{\text {rd }}$ Blink $\left(t_{6}\right)$ & 4.38 & 8.57 & -4.89 & 23.32 & $13.79 \pm 16.35$ & \\
\hline & $4^{\text {th }}$ Blink $\left(t_{8}\right)$ & 17.37 & 14.33 & 24.18 & 5.78 & $17.71 \pm 15.70$ & \\
\hline & $5^{\text {th }}$ Blink $\left(t_{10}\right)$ & 2.09 & -14.75 & 19.51 & 25.90 & $17.68 \pm 19.13$ & \\
\hline
\end{tabular}

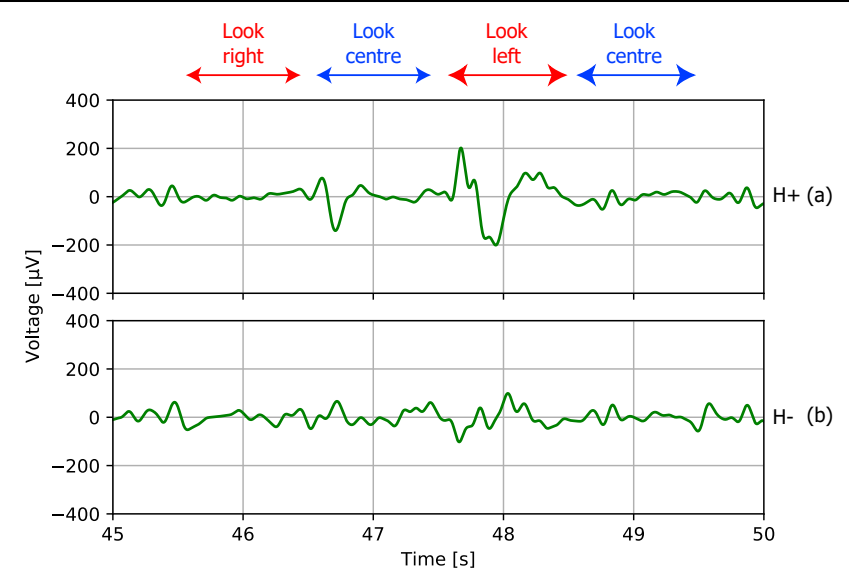

Fig. 4. Sample data from mask B showing the signals from each electrode for a smooth pursuit movement: right $\rightarrow$ left $\rightarrow$ centre.

the $\mathrm{H}+$ and $\mathrm{H}$ - electrode, while a participant performs this smooth pursuit eye movement. In this event the signals are much larger than the noise floor and can clearly be seen by eye. In this record the noise floor was $V_{\text {noise }}=77 \mu V_{r m s}$. This is in contrast to another example record where the noise floor was $V_{\text {noise }}=435 \mu V_{r m s}$, a high amount of noise that dominates over the signal from the eye movement. The high noise floor in this example includes noise from the participant performing a number of unwanted movements, such as involuntary eye blinks and motion artifacts, as well as low level background noise. In this example it is not possible to detect this type of eye movement.

Overall, half of the records had too high a noise floor to detect eye movements other than eye blinks. This is a result of both poor fitting on these participants (from variations in head diameters) and participants having difficulty reducing involuntary blinks leading to a higher reported noise floor. In this paper we have only used bandpass filtering for processing the data, further processing to remove other artifacts in the EOG signal could significantly improve the SNR.

\section{CONCLUSIONS}

We have reported on the performance of using textile graphene-based electrodes for performing EOG recordings with these electrodes sewn in to a conventional sleep mask. We have shown that this system can be used for EOG measurements, and can reliably detect eye blinks with a high SNR of over $16 \mathrm{~dB}$. We have also shown that we can, in some cases, detect smooth pursuit eye movements. It was found that in many cases it was difficult to detect the smooth pursuit eye movements, likely owing to the variation in tightness of the mask strap between participants from different head diameters. However, these electrodes are only held in place with a standard elastic strap, as opposed to traditional electrodes that are held on with an adhesive pad, giving our mask better comfort and ease of use. The results indicate that this material may be suitable for the tracking of eye movements in REM sleep in a far more comfortable form factor than conventional systems.

\section{REFERENCES}

[1] J. A. Groeger, F. R. H. Zijlstra, and D.-J. Dijk, "Sleep quantity, sleep difficulties and their perceived consequences in a representative sample of some 2000 british adults," $J$. Sleep Res., vol. 13, no. 4, pp. 359-371, 2004.

[2] T. Young, P. E. Peppard, and D. J. Gottlieb, "Epidemiology of obstructive sleep apnea," Am. J. Respir. Crit. Care Med., vol. 165, no. 9, pp. 1217-1239, 2002.

[3] P. Maquet, J.-M. Péters, J. Aerts, et al., "Functional neuroanatomy of human rapid-eye-movement sleep and dreaming," Nature, vol. 383, no. 6596, pp. 163-166, 1996.

[4] R. Stickgold, "Sleep-dependent memory consolidation," $\mathrm{Na}$ ture, vol. 437, no. 7063, pp. 1272-1278, 2005.

[5] D.-W. Chang, Y.-D. Liu, C.-P. Young, et al., "Design and implementation of a modularized polysomnography system," IEEE Trans. Instrum. and Meas., vol. 61, no. 7, pp. 19331944, 2012.

[6] S. Liang, C. Kuo, Y. Lee, et al., "Development of an EOGbased automatic sleep-monitoring eye mask," IEEE Trans. Instrum. and Meas., vol. 64, no. 11, pp. 2977-2985, 2015.

[7] A. J. Golparvar and M. K. Yapici, "Electrooculography by wearable graphene textiles," IEEE Sens. J., vol. 18, no. 21, pp. 8971-8978, 2018.

[8] N. Karim, S. Afroj, A. Malandraki, et al., "All inkjetprinted graphene-based conductive patterns for wearable etextile applications," J. Mater. Chem. C, vol. 5, no. 44, pp. $11640-11648,2017$.

[9] C. Beach, N. Karim, and A. J. Casson, "Performance of graphene ECG electrodes under varying conditions," in IEEE EMBC, Honolulu, Jul. 2018.

[10] N. Karim, S. Afroj, S. Tan, et al., "Scalable production of graphene-based wearable e-textiles," ACS Nano, vol. 11, no. 12, pp. 12 266-12 275, 2017.

[11] S. Afroj, N. Karim, Z. Wang, et al., "Engineering graphene flakes for wearable textile sensors via highly scalable and ultrafast yarn dyeing technique," ACS Nano, vol. 13, no. 4, pp. 3847-3857, 2019.

[12] J. Virkkala, J. Hasan, A. Värri, et al., "Automatic sleep stage classification using two-channel electro-oculography," J. Neurosci. Meth., vol. 166, no. 1, pp. 109-115, 2007. 